Pacific Journal of Mathematics 


\title{
CARDINALITY CONSTRAINTS FOR PSEUDOCOMPACT AND FOR TOTALLY DENSE SUBGROUPS OF COMPACT TOPOLOGICAL GROUPS
}

\author{
W. W. COMFort AND Lewis C. ROBERTSON
}

\begin{abstract}
Let $K$ be a compact, Hausdorff topological group, $\mathscr{G}(K)$ the set of dense, pseudocompact subgroups of $K$, and $m(K)=\min \{|G|: G \in$ $\mathscr{G}(K)$ \}. We show: (1) $m(K)$ is a function of the weight of $K$ (in the sense that if $K^{\prime}$ is another such group with $w(K)=w\left(K^{\prime}\right)$, then $m(K)=m\left(K^{\prime}\right)$ ); and (2) if $K$ is connected then every totally dense subgroup $D$ of $K$ satisfies $|D|=|K|$. With these results in hand we classify (a) those cardinals $\alpha$ such that $m(K)<|K|$ when $w(K)=\alpha$ and (b) those cardinals $\alpha$ such that some compact $K$ with $w(K)=\alpha$ admits a totally dense subgroup $D$ with $|D|<|K|$. The conditions of (a) and (b) are incompatible in some models of ZFC (e.g., under GCH) and are compatible in others. Thus the following question, the origin of this work, is undecidable in ZFC: Is there a compact, Hausdorff, topological group $K$ with a totally dense, pseudocompact subgroup $G$ such that $|G|<|K|$ ?
\end{abstract}

1. Notation and conventions. We denote the least infinite cardinal number by the symbol $\omega$.

Let $\alpha$ be an infinite cardinal. We denote by $\alpha^{+}$the least cardinal $\beta$ such that $\beta>\alpha$, and we denote by $\operatorname{cf}(\alpha)$ the least cardinal $\gamma$ such that there exists a family $\left\{\alpha_{i}: i \in I\right\}$ with $|I|=\gamma$ for which each $\alpha_{i}<\alpha$ and $\sum_{i \in I} \alpha_{i}=\alpha$.

The symbols $\mathbf{Z}, \mathbf{Q}, \mathbf{R}$ and $\mathbf{T}$ denote respectively the sets of integers, of rational numbers, of real numbers, and of complex numbers of modulus 1 . In each case we assume when convenient the usual algebraic and topological properties.

By a space, or a topological space, we mean a completely regular, Hausdorff space, i.e., a Tychonoff space. By a topological group we mean an ordered triple $G=\langle G, \circ, \mathscr{T}\rangle$ such that

(i) $\langle G, \circ\rangle$ is a group,

(ii) $\langle G, \mathscr{T}\rangle$ is a topological space, and

(iii) the function $\langle x, y\rangle \rightarrow x y^{-1}$ is continuous from $G \times G$ to $G$.

It is well known (see for example [28] (8.4)) that if $\langle G, \circ, \mathscr{T}\rangle$ satisfies (i) and (iii) and the $T_{0}$ separation property, then $\langle G, \mathscr{T}\rangle$ is a Tychonoff space. 
For a space $X=\langle X, \mathscr{T}\rangle$, we denote by $P X$ or by $P(X)$ the set $X$ with the smallest topology $\mathscr{T}^{\prime}$ such that $\mathscr{T}^{\prime} \supset \mathscr{T}$ and every $G_{\delta}$ of $\mathscr{T}^{\prime}$ is $\mathscr{T}^{\prime}$-open; the topology $\mathscr{T}^{\prime}$ is defined by the requirement that

$$
\{\cap \mathscr{U}: \mathscr{U} \subset \mathscr{T} \text { and }|\mathscr{U}| \leq \omega\}
$$

is a base for $\mathscr{T}^{\prime}$. It is not difficult to check (see for example [9] (page 31)) that when $\langle X, \mathscr{T}\rangle$ is given the pair $\left\langle X, \mathscr{T}^{\prime}\right\rangle$ is indeed a space, i.e., a completely regular, Hausdorff space. Among other notations used in the literature for what we here call $P X$ are $X_{\pi}$ [8], $X_{\omega^{+}}$[9], and $p X$ [55].

For a space $X=\langle X, \mathscr{T}\rangle$ we denote by $w(X)$ and $d(X)$ the weight and the density character of $X$, respectively.

Some of the results of this paper have been announced in [10], [6], [13] and [5].

2. Pseudocompact topological groups. A topological space $X$ is said to be pseudocompact if every real-valued continuous function on $X$ is bounded. It is a well-known result of Bagley, Connell and McKnight [2], exposed also by Engelking [18] (3.10.22), that $X$ is pseudocompact if and only if every locally finite family of open subsets of $X$ is finite. It was noted by Hewitt [27] in a fundamental paper in which inter alia pseudocompact spaces were first introduced and studied, that if a pseudocompact space $X$ is dense in a (Tychonoff) space $Y$, then $X$ is $G_{\delta}$-dense in $Y$ in the sense that $X$ meets every non-empty $G_{\delta}$ subset of $Y$.

A topological group $G$ is said to be totally bounded if for every nonempty, open subset $U$ of $G$ there is finite $F \subset G$ such that $G=F U$. It is a theorem of Weil [53] that every totally bounded group $G$ embeds as a dense subgroup of a compact group $\bar{G}$; further, $\bar{G}$ is unique in the sense that if $\tilde{G}$ is a compact group in which $G$ is dense then there is a function $\varphi$, simultaneously an algebraic isomorphism and a topological homeomorphism, from $\bar{G}$ onto $\tilde{G}$, such that $\varphi(x)=x$ for all $x \in G$. The group $\bar{G}$ is called the Weil completion of the (totally bounded) group $G$.

One checks easily [11] that if $G$ is a pseudocompact topological group then $G$ is totally bounded, i.e., that $\bar{G}$ is defined.

In the following theorem we collect from the literature several results needed below. Among the references cited the interested reader will find not only primary sources but also (i) related expository works and (ii) theorems strengthening the original statement(s), and arguments simpler than those given originally.

We give the statements of 2.1 in approximately their historical order. As has been remarked in [5] and elsewhere, however, the relatively recent results 2.1(c) and 2.1(d) combine to yield quick and easy proofs of 2.1(a) 
(as well as of other identities concerning cardinal numbers associated with compact topological groups).

For a cardinal number $\alpha \geq \omega$, the symbol $\log (\alpha)$ is defined by

$$
\log (\alpha)=\min \left\{\beta: 2^{\beta} \geq \alpha\right\}
$$

2.1. THEOREM. Let $K$ be a compact topological group such that $w(K)=$ $\alpha \geq \omega$, and let $G$ be a dense subgroup. (Thus $K=\bar{G}$.) Then

(a) ([37], [26], [33], [29] (28.58)) $|K|=2^{\alpha}$ and $d(K)=\log (\alpha)$.

(b) ([11], [51]) $G$ is pseudocompact if and only if $G$ is $G_{\delta^{-}}$-dense in $K$.

(c) $([11],[51],[16]) G$ is pseudocompact if and only if the Weil completion $K$ of $G$ is equal to the Stone-Čech compactification $\beta G$ of $G$.

(d) ([41], [34], [50], [28] $(25.35,9.15),[18](3.12 .12(b)))$ There is a continuous function from $\{0,1\}^{\alpha}$ onto $K$.

(e) ([45], [36] (3.18), [19], [20], [21], [3]) There is a continuous function from $K$ onto $[0,1]^{\alpha}$.

The following consequence of 2.1 is given in [12]. See also [31], [32], [56] and [57] for 2.2(b), and [44] (6.4) and [5] (7.6) for generalizations.

For a compact group $K$ the symbol $m(K)$ is defined as in the Abstract.

2.2. CoROllaRY. Let $K$ be a compact topological group with $w(K)=\alpha$ $\geq \omega$. Then

(a) $K$ contains a dense, countably compact subgroup $G$ such that $|G|=(\log (\alpha))^{\omega} ;$ and

(b) $m(K) \leq(\log (\alpha))^{\omega}$.

Proof. (a) For every subset $S$ of $K$ we define $S^{\prime}$ and [ $S$ ] as follows: $S^{\prime}$ is a set formed by adjoining to $S$ an accumulation point (in $K$ ) of each countably infinite subset of $S$, and [S] is the subgroup of $K$ generated by $S$. We note that $\left|S^{\prime}\right| \leq|S|^{\omega}$, and that $|[S]|=|S|$ if $|S| \geq \omega$.

From the Hewitt-Marczewski-Pondiczery theorem (cf. [18] (2.3.15)) there is a dense subset $D$ of $\{0,1\}^{\alpha}$ such that $|D| \leq \log (\alpha)$. Let $f$ be continuous from $\{0,1\}^{\alpha}$ onto $K$ and set $E=f[D]$; we have $|E| \leq \log (\alpha)$. From

$$
\log (\alpha) \leq(\log (\alpha))^{\omega} \leq \alpha^{\omega} \leq \alpha^{\alpha}=2^{\alpha}=|K|
$$

it follows that there is $F \subset K$ such that $F \supset E$ and $|F|=(\log (\alpha))^{\omega}$. For ordinals $\xi \leq \omega^{+}$we define subgroups $G_{\xi}$ of $K$ as follows: $G_{0}=[F]$, 
$G_{\xi+1}=\left[G_{\xi}^{\prime}\right]$ for $\xi<\omega^{+}$, and $G_{\xi}=\left[\left(\bigcup_{\eta<\xi} G_{\eta}\right)^{\prime}\right]$ for limit ordinals $\xi \leq \omega^{+}$. An easy induction shows $\left|G_{\xi}\right|=(\log (\alpha))^{\omega}$ for all $\xi \leq \omega^{+}$. The countably compact group $G=G_{\omega^{+}}$is then as required.

(b) Since every countably compact space is pseudocompact, this is a consequence of (a).

In 2.7 below we collect all the information known to us concerning the cardinal numbers $m(K)$ for compact topological groups $K$. With no claim for novelty, in the interest of completeness we include here the proofs of three lemmas which prepare the way.

We use the symbol $\approx$ to denote homeomorphism.

\subsection{LEMMA. If $\alpha$ is an infinite cardinal, then $P\left(\{0,1\}^{\alpha}\right) \approx P\left([0,1]^{\alpha}\right)$.}

Proof. Let $D$ be the discrete space such that $|D|=2^{\omega}$. For every space $X$ in which each point is a $G_{\delta}$ and $2 \leq|X| \leq 2^{\omega}$ we have $\left|X^{\omega}\right|=2^{\omega}$ and $P\left(X^{\omega}\right) \approx D$, so that

$$
P\left(X^{\alpha}\right) \approx P\left(\left(X^{\omega}\right)^{\alpha}\right) \approx P\left(\left(P\left(X^{\omega}\right)\right)^{\alpha}\right) \approx P\left(D^{\alpha}\right) .
$$

A topological space $X$ is said to be a Baire space if the countable intersection of dense, open subsets of $X$ is dense in $X$.

2.4. LeMMA. (a) If $X$ is a compact (Hausdorff) space, then $P X$ is a Baire space.

(b) $A G_{\delta}$-dense subspace of a Baire space is a Baire space.

Proof. (a) The set $\mathscr{B}$ of compact $G_{\delta}$-sets of $X$ is a base for the topology of $P X$. Let $B \in \mathscr{B}$ and let $\left\{U_{n}: n<\omega\right\}$ be a sequence of dense, open subsets of $P X$ with $U_{n} \supset U_{n+1}$. We show $\left(\bigcap_{n} U_{n}\right) \cap B \neq \varnothing$. Choose $x_{0} \in$ $B \cap U_{0}$ and then $B_{0} \in \mathscr{B}$ such that $x_{0} \in B_{0} \subset B \cap U_{0}$ and recursively, if $x_{k}$ and $B_{k}$ have been defined, choose $x_{k+1} \in B_{k} \cap U_{k+1}$ and then $B_{k+1} \in$ $\mathscr{B}$ so that $x_{k+1} \in B_{k+1} \subset B_{k} \cap U_{k+1}$; since each $B_{k}$ is $X$-compact we have $\varnothing \neq \bigcap_{n} B_{n} \subset\left(\cap_{n} U_{n}\right) \cap B$, as required.

(b) Let $Y$ be $G_{\delta}$-dense in the Baire space $X$, let $U$ be a non-empty open subset of $Y$ and let $U_{n}$ be dense and open in $Y$. There are open sets $\tilde{U}, \tilde{U}_{n}$ in $X$ such that $U=\tilde{U} \cap Y$ and $U_{n}=\tilde{U}_{n} \cap Y$. Since $X$ is Baire and $\tilde{U}_{n}$ is dense in $X$ we have $\left(\bigcap_{n} \tilde{U}_{n}\right) \cap \tilde{U} \neq \varnothing$, and since $Y$ is $G_{\delta}$-dense in $X$ we then have

$$
\varnothing \neq\left(\bigcap_{n} \tilde{U}_{n}\right) \cap \tilde{U} \cap Y=\left(\bigcap_{n} U_{n}\right) \cap U .
$$


2.5. LeMMA. Let $G$ be a non-discrete pseudocompact topological group. Then

(a) $|G| \geq 2^{\omega}$;

(b) $d(P G) \geq 2^{\omega}$; and

(c) $\operatorname{cf}(d(P G))>\omega$.

Proof. (a) Let $\lambda$ denote left Haar measure on the Weil completion $\bar{G}$ of $G$. Since $\lambda(\{e\})=0$ and $\lambda$ is regular, for $n<\omega$ there is a neighborhood $U_{n}$ in $\bar{G}$ of $e$ such that $\lambda\left(U_{n}\right)<1 / n$. According to a well-known theorem of Kakutani and Kodaira [38] (see [28] (8.7), [25] §64.G) or [5] (3.7) for expository treatments) there is a compact, normal, $G_{\delta}$ subgroup $N$ of $\bar{G}$ such that $N \subset \cap_{n} U_{n}$; the $\lambda$-measurable set $N$ satisfies $\lambda(N)=0$, so from $\lambda(\bar{G})=1$ follows $|\bar{G} / N|>\omega$. Since $\bar{G}$ is compact and $N$ is a $G_{\delta}$, the quotient $\bar{G} / N$ is metrizable. It follows that $|\bar{G} / N| \geq 2^{\omega}$. The group $G$ meets each coset of $N$ in $\bar{G}$ (by 2.1(b)), and (a) follows.

(b) Since $d(P G) \geq \omega$, there is a dense subgroup $H$ of $P G$ such that $|H|=d(P G)$. Now $H$ is $G_{\delta}$-dense in $G$, hence in $\bar{G}$. From $2.1(\mathrm{~b})$ it follows that $H$ is pseudocompact in the topology it inherits from $\bar{G}$, so that $|H| \geq 2^{\omega}$ by (a).

(c) The conclusion holds in case $d(P G)=2^{\omega}$, so we assume that $d(P G)>2^{\omega}$ and $\operatorname{cf}(d(P G))=\omega$. Let $D=\cup_{n} D_{n}$ be dense in $P G$ with $\omega \leq\left|D_{n}\right|<d(P G)$ for each $n$, and let $E_{n}$ be the closed subgroup of $P G$ generated by $D_{n}$. Now the topology of $P G$ is the topology inherited by $G$ from $P \bar{G}$, and the set $G$ is $G_{\delta}$-dense not only in $\bar{G}$ but in $P \bar{G}$ as well; it follows from 2.3 that $P G$ is a Baire space. Like every $F_{\sigma}$ in $P G$, the dense set $\cup_{n} E_{n}$ is closed in $P G$; thus $\cup_{n} E_{n}=P G$ and there is $n$ such that int $_{P G} E_{n} \neq \varnothing$. Since $E_{n}$ is then $P G$-open, there is a sequence $\left\{U_{k}: k<\omega\right\}$ of $\bar{G}$-open neighborhoods of $e$ such that $\left(\cap_{k} U_{k}\right) \cap G \subset E_{n}$. Again by the theorem of Kakutani and Kodaira [38] there is a closed, normal subgroup $N$ of $\bar{G}$ such that $N \subset \cap_{k} U_{k}$ and $\bar{G} / N$ is metrizable, and from

$$
\left|G / E_{n}\right| \leq|G /(N \cap G)| \leq|\bar{G} / N| \leq 2^{\omega}<d(P G)
$$

and $d\left(E_{n}\right)<d(P G)$ we have the contradiction

$$
d(P G) \leq d\left(E_{n}\right) \cdot\left|G / E_{n}\right|<d(P G) .
$$

2.6. REMARK. Here we make three comments concerning the question: "Does every infinite compact topological group $K$ contain a dense, pseudocompact subgroup $G$ such that $|G|<|K|$ ? (Restated: Is $m(K)<|K|$ for such $K$ ?) 
(a) It is not difficult to show (see for example [22] (3D)) that a pseudocompact, normal space is countably compact. Thus a pseudocompact metric space is compact. It follows that $m(K)=|K|$ for every compact metrizable group $K$.

(b) It is clear from $2.5(\mathrm{a})$ that if $2^{\omega}=2^{(\omega+)}$ then the compact group $K=\{-1,+1\}^{\left(\omega^{+}\right)}$satisfies $m(K)=|K|$. Indeed, as noted in [5], from 2.1(a) it follows that under this hypothesis the relation $m(K)=|K|$ holds for all compact groups $K$ with $w(K)=\omega^{+}$.

(c) it is a consequence of $2.7(\mathrm{e})$ below that compact groups $K$ with $m(K)=|K|$ can arise in circumstances unlike those of (a) and (b) - namely with $w(K)>\omega$ and in the presence of the generalized continuum hypothesis $(\mathrm{GCH})$. Indeed, it is enough to assume the so-called singular cardinals hypothesis $(\mathrm{SCH}): \kappa^{\lambda} \leq 2^{\lambda} \cdot \kappa^{+}$for all infinite cardinal numbers $\kappa$ and $\lambda$. The relevance of $\mathrm{SCH}$ to these topics came to our attention from Cater, Erdős and Galvin [4]; our 2.7(e) for $K=\{-1,+1\}^{\alpha}$ is a special case of their Theorem 2.5. Concerning this axiom they write (here we paraphrase slightly): "Clearly, $\mathrm{SCH}$ follows from the generalized continuum hypothesis, but is much weaker. In fact, models of set theory violating SCH are not easy to come by; Prikry and Silver (see Jech [35] (Section 37) and Magidor [42], [43]) have constructed such models assuming the consistency of very large (e.g., supercompact) cardinals, and Devlin and Jensen [14] have shown that some large cardinal assumption is necessary."

In order that $2.7(\mathrm{e})$ be not vacuous or nugatory, it is necessary to know that the existence of uncountable cardinals $\alpha$ such that $(\log (\alpha))^{\omega} \neq$ $2^{\alpha}$ is consistent with $\mathrm{SCH}$. In $\S 3$ we characterize such cardinals $\alpha$ and we show that they exist in profusion in all models of ZFC.

2.7. THEOREM. Let $K$ and $K^{\prime}$ be compact topological groups with $w(K)=w\left(K^{\prime}\right)=\alpha \geq \omega$. Then

(a) $m(K)=m\left(K^{\prime}\right)$ - that is, the cardinal $m(K)$ depends only on the cardinal $\alpha=w(K)$, not on the algebraic structure of $K$.

(b) $m(K) \geq 2^{\omega}$ and $\operatorname{cf}(m(K))>\omega$.

(c) $\log (\alpha) \leq m(K) \leq(\log \alpha)^{\omega}$.

(d) If $(\log \alpha)^{\omega}<2^{\alpha}$, then $m(K)<|K|$.

(e) Assume SCH. Then $m(K)=(\log (\alpha))^{\omega}$ (and hence $m(K)=|K|$ if $\left.(\log (\alpha))^{\omega}=2^{\alpha}\right)$.

Proof. By 2.1(d) and 2.1(e) there are continuous functions from $\{0,1\}^{\alpha}$ onto $K$ and from $K$ onto $[0,1]^{\alpha}$. Since the inverse image under a 
continuous surjection of a $G_{\delta}$ is a $G_{\delta}$, these functions remain continuous when $\{0,1\}^{\alpha}, K$ and $[0,1]^{\alpha}$ are replaced by $P\left(\{0,1\}^{\alpha}\right), P K$, and $P\left([0,1]^{\alpha}\right)$, respectively. Since $P\left(\{0,1\}^{\alpha}\right) \approx P\left([0,1]^{\alpha}\right)$ by 2.3 , it follows from elementary properties of continuous function sthat

$$
d\left(P\left(\{0,1\}^{\alpha}\right)\right)=d(P K)=d\left(P\left([0,1]^{\alpha}\right)\right) .
$$

Theorem 2.1(b) is essentially the statement that $m(K)=d(P K)$. Part (a) is now immediate; and from 2.5, so is part (b). The inequalities of (c) are given by 2.2 (b) and 2.1(a) (together with the observation that $d(K) \leq$ $d(P K)$ ), and (d) follows from (c) and 2.1(a). Thus it remains only to prove (e). We assume $m(K)<(\log (\alpha))^{\omega}$ and we consider two cases.

Case 1. $\log (\alpha)<2^{\omega}$. We then have the contradiction $2^{\omega} \leq m(K)<$ $(\log (\alpha))^{\omega} \leq 2^{\omega} \cdot(\log (\alpha))^{+}=2^{\omega}$.

Case 2. $\log (\alpha) \geq 2^{\omega}$. Then from

$$
\log (\alpha) \leq m(K)<(\log (\alpha))^{\omega} \leq 2^{\omega} \cdot(\log (\alpha))^{+}=(\log (\alpha))^{+}
$$

follows $m(K)=\log (\alpha)$ and hence $\operatorname{cf}(\log (\alpha))>\omega$. Then for every countable subset $A$ of $\log (\alpha)$ there is $\xi<\log (\alpha)$ such that $A \subset \xi$; hence

$$
\begin{aligned}
(\log (\alpha))^{\omega} & \leq \sum_{\xi<\log (\alpha)}|\xi|^{\omega} \leq \sum_{\xi<\log (\alpha)} 2^{\omega} \cdot|\xi|^{+} \\
& =\log (\alpha)<(\log (\alpha))^{\omega}
\end{aligned}
$$

a contradiction. The proof is complete.

2.8. Notation. For every cardinal $\alpha \geq \omega$, we set $m(\alpha)=$ $m\left(\{-1,+1\}^{\alpha}\right)$. (This cardinal is denoted $\Delta(\alpha, \omega)$ in [4].) According to 2.1(b) and 2.7(a) we have

$$
m(\alpha)=m(K)=d(P K)
$$

for every compact group $K$ with $w(K)=\alpha$. The condition that some (equivalently: every) compact group $K$ with $w(K)=\alpha$ contains a dense, pseudocompact subgroup $G$ such that $|G|<|K|$ is equivalent to the condition $m(\alpha)<2^{\alpha}$.

2.9. REMARKS. (a) While our statement and proof in 2.5(a) are probably those which are appropriate to a paper dealing with topological groups, the inequality $|X| \geq 2^{\omega}$ is valid for all infinite, homogeneous, pseudocompact spaces $X$. For a proof of this general statement and of 
other inequalities concerning cardinal invariants associated with homogeneous, pseudocompact spaces, see van Douwen [16].

(b) In the argument above concerning Theorem 2.7, we indicated that the space $P K$ is "caught between" the homeomorphic spaces $P\left(\{0,1\}^{\alpha}\right)$ and $P\left([0,1]^{\alpha}\right)$. Although the following conjecture, suggested by this situation, is probably of little importance or utility in its own right, we find mildly annoying our inability to prove or disprove it. Conjecture: If $K$ is a compact topological group with $w(K)=\alpha \geq \omega$, then $P K \approx P\left(\{0,1\}^{\alpha}\right)$.

(c) We know of no cardinal $\alpha$-in any model of ZFC - for which the relation $m(\alpha)=(\log (\alpha))^{\omega}$ fails.

3. Cardinals $\alpha$ such that $(\log (\alpha))^{\omega} \neq 2^{\alpha}$. We use the notation $\alpha_{n} \uparrow$ to indicate that $\left\{\alpha_{n}: n<\omega\right\}$ is a strictly increasing sequence of cardinal numbers; we write $\alpha_{n} \uparrow \alpha$ if $\alpha_{n} \uparrow$ and $\sum_{n<\omega} \alpha_{n}=\alpha$.

For ordinal numbers $\xi$, the Beth cardinals $\beth_{\xi}$ are defined as usual by the rules $\beth_{0}=\aleph_{0}=\omega, \beth_{\xi+1}=2^{\beth_{\xi}}$ for all $\xi$, and $\beth_{\xi}=\sum_{\zeta<\xi} \beth_{\zeta}$ for limit ordinals $\xi>0$. We note that the class of Beth cardinals is cofinal in the class of all cardinals. For $\alpha \geq \omega$ the least ordinal $\zeta$ such that $\beth_{\zeta}>\alpha$ cannot be a limit ordinal; hence for all $\alpha \geq \omega$ there is a (unique) ordinal $\xi$ such that $\beth_{\xi} \leq \alpha<\beth_{\xi+1}$.

We use below the fact that if $\xi$ is a positive limit ordinal then $\operatorname{cf}(\xi)=\operatorname{cf}\left(\beth_{\xi}\right)$. The proof of Tarski [48] is recorded also in [9] (1.25).

3.1. THEOREM. Let $\alpha$ be an infinite cardinal and let $\xi$ be the ordinal such that $\beth_{\xi} \leq \alpha<\beth_{\xi+1}$. Then the following conditions are equivalent.

(a) $(\log (\alpha))^{\omega}=2^{\alpha}$;

(b) (1) $2^{\alpha}=\beth_{\xi+1}$, and (2) either $\xi=0$, or $\xi$ is a positive limit ordinal such that $\operatorname{cf}(\xi)=\omega$.

Proof. (a) $\Rightarrow$ (b). (1) Clearly $2^{\alpha} \geq \beth_{\xi+1}$. If $2^{\alpha}>\beth_{\xi+1}$ then from $\log (\alpha)$ $\leq \beth_{\xi}$ follows the contradiction

$$
\beth_{\xi+1}<2^{\alpha}=(\log (\alpha))^{\omega} \leq \beth_{\xi}^{\omega} \leq 2^{\beth_{\xi}}=\beth_{\xi+1} .
$$

(2) If there is $\zeta$ such that $\xi=\zeta+1$ we have the contradiction

$$
\beth_{\xi+1}=2^{\alpha}=(\log (\alpha))^{\omega} \leq \beth_{\xi}^{\omega}=2^{Z_{\xi} \cdot \omega}=2^{Z_{\xi}}=\beth_{\xi} .
$$

Thus either $\xi=0$ or $\xi$ is a positive limit ordinal. Suppose now that $\xi>0$ and $\operatorname{cf}(\xi)>\omega$. Since $\operatorname{cf}\left(\beth_{\xi}\right)>\omega$ and $\beth_{\xi}=\sup _{\zeta<\xi} \beth_{\zeta}$, for every countable $A \subset \beth_{\xi}$ there is $\zeta<\xi$ such that $A \subset \beth_{\xi}$; this yields the contradiction

$$
\beth_{\xi+1}=(\log (\alpha))^{\omega} \leq \beth_{\xi}^{\omega} \leq \sum_{\zeta<\xi} \beth_{\zeta}^{\omega} \leq \sum_{\zeta<\xi} 2^{\beth_{\xi}}=\beth_{\xi} .
$$


(b) $\Rightarrow$ (a). It is enough to prove (i) $\beth_{\xi}^{\omega}=\beth_{\xi+1}$ and (ii) $\log (\alpha)=\beth_{\xi}$. For $\xi=0$ both statements are clear so we assume that $\xi$ is a positive limit ordinal with $\operatorname{cf}(\xi)=\omega$ and, using $\operatorname{cf}\left(\beth_{\xi}\right)=\omega$, we choose $\beta_{n} \uparrow \beth_{\xi}$. For (i) we write

$$
\beth_{\xi+1}=2^{\beth_{\xi}}=2^{\Sigma \beta_{n}}=\prod 2^{\beta_{n}} \leq \beth_{\xi}^{\omega} \leq 2^{\beth_{\xi}}=\beth_{\xi+1},
$$

and we note that if (ii) fails then there is $\zeta<\xi$ such that $\log (\alpha)<\beth_{\zeta}$ and we have the contradiction

$$
\alpha \leq 2^{\log (\alpha)} \leq 2^{\beth_{\zeta}}=\beth_{\zeta+1}<\beth_{\xi} \leq \alpha .
$$

The proof is complete.

We emphasize that $3.2(a)$, a consequence of 3.1 , is valid in every model of ZFC.

3.2. Let $\beta$ be an infinite cardinal.

(a) There is a limit cardinal $\alpha \geq \beta$ such that $m(\alpha)<2^{\alpha}$.

(b) Assume SCH. There is a limit cardinal $\alpha \geq \beta$ such that $m(\alpha)=2^{\alpha}$.

Proof. (a) By 2.7(d) it is enough to choose $\alpha \geq \beta$ such that $(\log (\alpha))^{\omega}$ $<2^{\alpha}$ and for this, according to 3.1 , it is sufficient to take $\alpha=\beth_{\xi} \geq \beta$ with $\xi$ a positive limit ordinal such that $\operatorname{cf}(\xi)>\omega$.

(If the condition that $\alpha$ be a limit cardinal is omitted, the cardinal $\alpha=\beta^{\omega}$ is as required. For in this case one has $m(\alpha) \leq(\log (\alpha))^{\omega} \leq \alpha^{\omega}=$ $\alpha<2^{\alpha}$.)

(b) Set $\alpha_{0}=\beta$, recursively for $n<\omega$ define $\alpha_{n+1}=2^{\alpha_{n}}$, and set $\alpha=\sup _{n} \alpha_{n}$. Then $\alpha=\log (\alpha)$ and

$$
2^{\alpha}=2^{\sum \alpha_{n}}=\prod 2^{\alpha_{n}} \leq \alpha^{\omega} \leq 2^{\alpha},
$$

so $(\log (\alpha))^{\omega}=2^{\alpha}$; the required conclusion then follows from $2.7(\mathrm{e})$.

3.3. The infinite compact groups $K$ which are metrizable are exactly those for which $w(K)=\omega$. We have remarked in 2.6(a) in effect that $m(\omega)=2^{\omega}$. We do not know whether the conclusion of 3.2(b) remains valid when the hypothesis $\mathrm{SCH}$ is omitted; we do not even know whether there is in every model of ZFC an uncountable cardinal $\alpha$ such that $m(\alpha)=2^{\alpha}$.

4. Connected groups and products of simple groups. For $G$ a group, we denote by $Z(G)$ the center of $G$. In this section we combine an elegant result of van der Waerden [52] with a structure theorem of Weil [54] to 
sharpen the following structure theorem of Varopoulos [49] (4.1): If $G$ is compact and connected then the quotient $G / Z(G)$ is (topologically isomorphic to) a product $\Pi_{i} G_{i}$ with each $G_{i}$ metrizable. Our result, given in 4.2 , is used in 5.6 below in our study of totally dense subgroups.

The symbol $\simeq$ may be read "is topologically isomorphic to".

For $G$ a group and $N$ a normal subgroup of $G$, we denote by $\varphi_{N}$ the canonical homomorphism of $G$ onto $G / N$.

4.1. LeMMA. Let $G$ be a compact, connected group such that $\{e\}$ is the only normal Abelian subgroup of $G / Z(G)$, and let $N$ be a closed, normal, totally disconnected subgroup of $G$. Then the quotient group $K=G / N$ has the property that $K / Z(K) \simeq G / Z(G)$.

Proof. For $a \in N$ the function $G \rightarrow N$ given by $x \rightarrow x a x^{-1}$ has connected range containing the point $a$. Thus $x a x^{-1}=a$ for all $\langle a, x\rangle \in$ $N \times G$ and we have $N \subset Z(G)$. With $H=\varphi_{N}[Z(G)]$ we have

$$
\begin{aligned}
K / H & \simeq(G / N) /(Z(G) /(Z(G) \cap N)) \\
& =(G / N) /(Z(G) / N) \simeq G / Z(G),
\end{aligned}
$$

so it is enough to show $H=Z(K)$. The inclusion $\subset$ is clear. The function $\varphi_{H}: K \rightarrow K / H$ takes $Z(K)$ to a normal, Abelian subgroup of $K / H$, so from $K / H \simeq G / Z(G)$ follows $\varphi_{H}[Z(K)]=\{e\}$ and hence $Z(K) \subset H$, as required.

The following statement may be compared with the result cited above of Varopoulos [49].

4.2. THEOREM. Let $K$ be a compact, connected group. Then $K / Z(K)$ has the form $K / Z(K) \simeq \prod_{i \in I} H_{i}$ where each $H_{i}$ is a compact, connected, non-Abelian Lie group which is (algebraically) simple.

Proof. According to the structure theorem of Weil [54] (pp. 89-91) there are a compact group $G=A \times \Sigma^{*}$ and a continuous homomorphism $\psi$ from $G$ onto $K$ such that

(i) $A$ is a compact, connected Abelian group,

(ii) ker $\psi$ is a totally disconnected subgroup of $A \times Z\left(\Sigma^{*}\right)$, and

(iii) $\Sigma^{*}$ is a product of the form $\Sigma^{*}=\prod_{i \in I} G_{i}$ with each $G_{i}$ a compact, connected, simply connected, non-Abelian Lie group with a simple Lie algebra and a finite center.

We note that

$$
G / Z(G)=\left(A \times \Sigma^{*}\right) / A \times Z\left(\Sigma^{*}\right) \simeq \Sigma^{*} / Z\left(\Sigma^{*}\right),
$$


the "cancellation of $A$ " being justified by the fact that the natural surjective homomorphism sequence

$$
A \times \Sigma^{*} \rightarrow \Sigma^{*} \rightarrow \Sigma^{*} / Z\left(\Sigma^{*}\right)
$$

has kernel $A \times Z\left(\Sigma^{*}\right)$.

We set $H_{i}=G_{i} / Z\left(G_{i}\right)$. It is a theorem of van der Waerden [52] that the connected Lie groups $H_{i}$ are algebraically simple. (In particular, each is non-Abelian.) It is clear that $Z\left(\Sigma^{*}\right)=\prod_{i} Z\left(G_{i}\right)$. Every normal Abelian subgroup $S$ of $\Pi_{i} H_{i}$ satisfies $|S|=1$, since otherwise there is $i$ such that $\pi_{i}[S]$ is a non-trivial normal Abelian subgroup of $H_{i}$. The group $G$ then satisfies the hypotheses of 4.1 , and (taking $N=\operatorname{ker} \psi$ there) we have

$$
K / Z(K) \simeq G / Z(G) \simeq \Sigma^{*} / Z\left(\Sigma^{*}\right) \simeq \prod_{i \in I} H_{i},
$$

as required.

For a topological group $G$ we denote by $C(G)$ the component of the identity in $G$.

4.3. Corollary. Let $K$ be a compact, connected group. If $w(C(Z(K)))$ $<w(K)$, then $w(K / Z(K))=w(K)$.

Proof. Suppose first that $w(K)=\omega$. If $K$ is Abelian then $C(Z(K))=$ $K$, and if $K$ is non-Abelian then $K / Z(K)$ is infinite (so that $\omega \leq$ $w(K / Z(K)) \leq w(K)=\omega)$. We assume therefore in what follows that $w(K)>\omega$, we retain the notation of the proof of 4.2 , and we assume without loss of generality, replacing if necessary $A \times\{e\}$ by $A \times$ $\{e\} /(\operatorname{ker} \psi)$, that $\psi$ is an isomorphism on $A \times\{e\}$.

From $A \subset C(Z(G))$ follows $\psi[A] \subset C(Z(K))$. Hence $w(A)<w\left(\Sigma^{*}\right)$, since otherwise we have the contradiction

$$
w(K) \leq w\left(A \times \Sigma^{*}\right)=w(A)=w(\psi[A]) \leq w(C(Z(K)))<w(K) .
$$

But then from

$$
\omega<w(K) \leq w\left(A \times \Sigma^{*}\right)=w\left(\Sigma^{*}\right)=w\left(\prod_{i \in I} G_{i}\right)
$$

and the fact that $w\left(G_{i}\right)=\omega$ for each $i \in I$ follows $|I|=w\left(\Pi_{i \in I} G_{i}\right)$, so that

$$
w(K) \leq|I|=w\left(\prod_{i \in I} H_{i}\right)=w(K / Z(K)) \leq w(K),
$$

as required.

5. Totally dense subgroups. A subgroup $G$ of a topological group $K$ is said to be totally dense (in $K$ ) if, for each closed subgroup $H$ of $K$, the set $G \cap H$ is dense in $H$. Of course, every totally dense subgroup is dense. 
The concept has been studied in its own right [46], [24], [13], [39], and in connection with the problem of characterizing those topological groups $\langle G, \mathscr{T}\rangle$ for which $\mathscr{T}$ is minimal among (Hausdorff) topologies making $G$ a topological group [47], [23].

5.1. Notation. For $K$ a group, we denote by $t(K)$ the subgroup of $K$ generated by the set of torsion elements of $K$.

In a topological group $K$, every $x \in t(K)$ belongs to a finite subgroup $H(x)$ of $K$. Since $H(x)$ has no proper dense subsets, every totally dense subgroup $G$ of $K$ satisfies $x \in H(x) \subset G$. This observation proves the following simple statement.

5.2. LEMMA. If $K$ is a topological group and $G$ is totally dense in $K$, then $G \supset t(K)$.

We saw in 2.7(a) that the question of the minimal cardinality of a dense, pseudocompact subgroup of a compact group $K$ depends only on the cardinal number $w(K)$ and is quite insensitive to algebraic properties of $K$. As Lemma 5.2 suggests, and as will become clear in Theorem 5.8 below, the analogous cardinality question concerning totally dense subgroups commands a very different kind of response: For every $\alpha \geq \omega$ there are compact groups $K$ with $w(K)=\alpha$ such that $K$ has no proper totally dense subgroup. Indeed, as 5.2 makes clear, $\{-1,+1\}^{\alpha}$ is such a group.

Let us say for simplicity that a subgroup $G$ of a group $K$ is small if $|G|<|K|$. Our objective in this section is to classify those cardinal numbers $\alpha$ for which some compact group $K$ with $w(K)=\alpha$ contains a small, totally dense subgroup. (We show inter alia that such a group $K$, when it exists, may be chosen totally disconnected and Abelian.)

5.3. LEMMA. Let $K$ be a compact group and $G$ a totally dense subgroup. If $\varphi$ is a continuous homomorphism from $K$ onto a (compact) group $K^{\prime}$, then $\varphi[G]$ is totally dense in $K^{\prime}$.

Proof. If $H^{\prime}$ is a closed subgroup of $K^{\prime}$ then $G \cap \varphi^{-1}\left(H^{\prime}\right)$ is dense in $\varphi^{-1}\left(H^{\prime}\right)$; hence $\varphi[G]$ is dense in $H^{\prime}$.

Next we characterize in set-theoretic terms those cardinals $\alpha$ which, according to 5.8 below, have the form $\alpha=w(K)$ for some compact group $K$ with a small totally dense subgroup. We extend the list beyond the 
demands of strict economy and the requirements of 5.8 in the belief that the indicated equivalences are of independent interest.

It is a well-known theorem of König [40], for which proofs are recorded (for example) in [1] and [9] (1.19), that if $I$ is an index set and cardinal families $\left\{a_{i}: i \in I\right\}$ and $\left\{b_{i}: i \in I\right\}$ satisfy $a_{i}<b_{i}$ for all $i \in I$, then $\sum_{i} a_{i}<\Pi_{i} b_{i}$. In what follows we use two immediate consequences of König's theorem: If $\alpha$ is an infinite cardinal number then $\alpha^{\operatorname{cf}(\alpha)}>\alpha$ and (hence) $\operatorname{cf}\left(2^{\alpha}\right)>\alpha$.

5.4. LEMMA. Let $\alpha$ be an infinite cardinal such that $\operatorname{cf}(\alpha)=\omega$, let $\left\{\alpha_{n}\right.$ : $n<\omega\}$ be any set of distinct cardinals such that $\alpha_{n}<\alpha($ all $n)$ and $\sum \alpha_{n}=\alpha$, and define $\beta=\sum 2^{\alpha_{n}}$. Then the following conditions are equivalent.

(a) $\alpha=\log \left(2^{\alpha}\right)$

(b) $2^{\alpha_{n}}<2^{\alpha}($ all $n<\omega)$;

(c) $2^{\alpha_{n}}<\beta($ all $n<\omega)$;

(d) there is a sequence $n(k)$ such that $2^{\alpha_{n(k)} \uparrow \beta \text {; }}$

(e) $\left\{2^{\alpha_{n}}: n<\omega\right\}$ has no largest element; and

(f) $\beta<2^{\alpha}$.

Proof. The conditions are all true, hence equivalent, if $\alpha=\omega$. We assume therefore that $\alpha>\omega$ and that $\alpha_{n} \geq \omega$ for all $n<\omega$.

(a) $\Rightarrow$ (b). This is obvious.

(b) $\Rightarrow$ (c). If (c) fails then $\beta<2^{\alpha}$ and $\beta^{\omega}=\beta$. But

$$
2^{\alpha}=2^{\Sigma_{n} \alpha_{n}}=\prod_{n} 2^{\alpha_{n}} \leq \prod_{n} \beta=\beta^{\omega} \leq\left(2^{\alpha}\right)^{\omega}=2^{\alpha}
$$

and hence $\beta=\beta^{\omega}=2^{\alpha}$.

(c) $\Rightarrow$ (d). From $2^{\alpha_{n}}<\beta$ and $\beta=\sum 2^{\alpha_{n}}$ follows $\operatorname{cf}(\beta)=\omega$ so if (d) fails then there is $\gamma<\beta$ such that $2^{\alpha_{n}} \leq \gamma$ for all $n<\omega$. But then

$$
\beta=\sum_{n} 2^{\alpha_{n}} \leq \sum_{n} \gamma=\omega \cdot \gamma<\beta \text {. }
$$

(d) $\Rightarrow$ (e). From (d) follows $\operatorname{cf}(\beta)=\omega$ and hence $\beta<\beta^{\omega}$. It follows that if there is $N<\omega$ such that $2^{\alpha_{N}} \geq 2^{\alpha_{n}}$ for all $n$, then $2^{\alpha_{N}}<\beta$. But then

$$
\beta=\sum_{n} 2^{\alpha_{n}} \leq \sum_{n} 2^{\alpha_{N}}=\omega \cdot 2^{\alpha_{N}}<\beta \text {. }
$$

(e) $\Rightarrow$ (f). From (e) follows $2^{\alpha_{n}}<\beta$ for all $n<\omega$, for otherwise there are $m, n<\omega$ such that

$$
\beta \geq 2^{\alpha_{m}}>2^{\alpha_{n}} \geq \beta
$$

Thus again $\operatorname{cf}(\beta)=\omega$, while if (f) fails then $\operatorname{cf}(\beta)>\alpha$. 
(f) $\Rightarrow$ (a). If there is $\gamma<\alpha$ such that $2^{\gamma} \geq 2^{\alpha}$ then from $\alpha_{n}<\alpha$ and $\alpha=\sum \alpha_{n}$ it follows that there is $n$ such that $\gamma<\alpha_{n}<\alpha$. But then

$$
2^{\alpha} \leq 2^{\gamma} \leq 2^{\alpha_{n}} \leq \beta<2^{\alpha} \text {. }
$$

5.5. REMARKS. (a) When the conditions of 5.4 are satisfied we have

$$
2^{\alpha}=2^{\Sigma \alpha_{n}}=\prod 2^{\alpha_{n}} \leq\left(2^{\alpha}\right)^{\omega}=2^{\alpha}
$$

and hence $2^{\alpha}=\Pi 2^{\alpha_{n}}$.

(b) The conditions of 5.4 are in addition clearly equivalent to the condition $\alpha=\log (\beta)$.

(c) With focus on condition 5.4(b), we say that for cardinals $\alpha$ as in 5.4 the function $\gamma \rightarrow 2^{\gamma}$ is "discontinuous at $\alpha$ ".

It was noted in [13] that a compact, connected Abelian group $K$ with $w(K)=\alpha>\omega$ can have no small totally dense subgroup. (Proof. There is [13] a continuous homomorphism $\varphi$ from $K$ onto $\mathbf{T}^{\alpha}$, and since $t\left(\mathbf{T}^{\alpha}\right) \supset$ $\{-1,+1\}^{\alpha}$ any totally dense subgroup $G$ of $K$ must satisfy

$$
\left.|G| \geq|\varphi[G]| \geq\left|t\left(\mathbf{T}^{\alpha}\right)\right| \geq\left|\{-1,+1\}^{\alpha}\right|=2^{\alpha}=|K| .\right)
$$

The following theorem strengthens this result by removing the hypothesis that $K$ is Abelian.

5.6. THEOREM. Let $K$ be a compact, connected group with $w(K)=\alpha>$ $\omega$. Then $K$ has no small totally dense subgroup.

Proof. Suppose that $G$ is a small totally dense subgroup of $K$. Since $G \cap C(Z(K))$ is totally dense in the connected, Abelian group $C(Z(K))$ we have $w(C(Z(K)))<\alpha$ by the result cited just above from [13] (for otherwise

$$
\left.|G \cap C(Z(K))| \leq|G|<|K|=2^{\alpha}=|C(Z(K))|\right) .
$$

Hence from 4.3 and 4.2 we have $w(K / Z(K))=\alpha$ and $K / Z(K) \simeq \prod_{i \in I} H_{i}$ with each $H_{i}$ a compact, connected, non-Abelian Lie group. As is wellknown, each such group $H_{i}$ contains a non-degenerate compact, connected Abelian subgroup $A_{i}$. (For example, according to Hochschild [30] (p. 150 ff.) every $x \in H_{i}$ lies along a one-parameter subgroup, i.e., there is a continuous homomorphism $f: \mathbf{R} \rightarrow H_{i}$ such that $f(1)=x$. One may take $A_{i}=\operatorname{cl}_{H_{t}} f[\mathbf{R}]$; such a choice of $A_{t}$ is in fact a topological subgroup of $H_{i}$ isomorphic to a torus $\mathrm{T}^{n}$ with $0<n<\omega$.) We set $A=\prod_{i \in I} A_{i}$. According to 5.3 the homomorphism $\varphi: K \rightarrow K / Z(K)$ takes $G$ onto a totally dense subgroup of $K / Z(K)$, and again from $w\left(\prod_{i \in I} H_{i}\right)=\alpha>\omega$ and 
each $w\left(H_{i}\right)=\omega$ we have $|I|=\alpha$ and hence

$$
|\varphi[G] \cap A| \leq|G|<|K|=2^{\alpha}=|A| .
$$

We have: $A$ is a compact, connected Abelian group; $w(A)=\alpha>\omega$; and $\varphi[G] \cap A$ is a small totally dense subgroup of $A$. This contradiction completes the proof.

5.7. REMARK. It is interesting to notice (though logically inessential to our principal concern) that for non-Abelian groups the conclusion of 5.6 is valid even when $\alpha=\omega$. Specifically, we have

(a) there exist compact, connected, Abelian metrizable groups with a small totally dense subgroup, and

(b) there is no compact, connected, non-Abelian metrizable group with a small totally dense subgroup.

For (a), it is enough to consider the topological groups $\mathbf{T}^{n}$ for $0<n<\omega$. To prove (b), let $G$ be a totally dense subgroup of a compact, connected, non-Abelian metrizable group $K$ and write $K / Z(K)=\prod_{i \in I} H_{i}$ as in 4.2. With $\varphi: K \rightarrow K / Z(K)$ the canonical homomorphism and $\pi_{i}$ : $K / Z(K) \rightarrow H_{i}$ the projection, the group $\pi_{i}^{\circ} \varphi[G]$ is totally dense in $H_{i}$. Thus to prove $|G|=|K|=2^{\omega}$ it is enough to show that every totally dense subgroup $D$ of a compact, connected, non-Abelian, metrizable, algebraically simple Lie group $H$ satisfies $|D|=|H|=2^{\omega}$. We have noted already in the proof of 5.6, with Hochschild [30] (p. 151), that $H$ contains a topological subgroup isomorphic to $\mathbf{T}$. Thus there is $x \in H$ such that $x \neq e$ and $x^{2}=e$. Since the subgroup $S=\{x, e\}$ is not normal in $H$, its normalizer $N(S)$ is not equal to $H$; thus the (compact, connected) coset space $H / N(S)$ satisfies $|H / N(S)|=2^{\omega}$. Now $h S h^{-1} \cap k S k^{-1}=\{e\}$ whenever $h, k \in H$ and $h N(S) \neq k N(S)$, and $D$ contains each of the subgroups $h S h^{-1}$. Thus

$$
|D| \geq|H / N(S)|=2^{\omega},
$$

as required.

5.8. THEOREM. For each infinite cardinal number $\alpha$, the following conditions are equivalent.

(a) $\operatorname{cf}(\alpha)=\omega$ and $\alpha=\log \left(2^{\alpha}\right)$.

(b) Some compact, totally disconnected group $K$ with $w(K)=\alpha$ has a small, totally dense subgroup.

(c) Some compact group $K$ with $w(K)=\alpha$ has a small, totally dense subgroup. 
Proof. We show (a) $\Leftrightarrow$ (b) and (b) $\Leftrightarrow$ (c).

(a) $\Rightarrow$ (b). Here we show that in fact $K$ may be chosen Abelian. Let $\left\{p_{n}: n<\omega\right\}$ be an enumeration of the positive primes in their usual order: $p_{0}=2, p_{1}=3, \ldots$ Using 5.4 we choose $\alpha_{n} \uparrow \alpha$ such that $2^{\alpha_{n}} \uparrow$, and we set $K(n)=Z\left(p_{n}\right)^{\alpha_{n}}, K=\Pi_{n} K(n)$, and $G=\oplus_{n} K(n)$. Since $2^{\alpha_{n}} \uparrow$ we have from 5.4 or directly from König's theorem that

$$
|G|=\sum 2^{\alpha_{n}}<\prod 2^{\alpha_{n}}=\prod|K(n)|=|K| .
$$

The other assertions of (b) being now obvious, let us summarize from [13] (5.5) the proof that $G$ is totally dense in $K$. Since $G=t(K)$ it is enough to show for every closed subgroup $H$ of $K$ that $t(H)$, which is $G \cap H$, is dense in $H$. The dual group $\hat{K}$ of $K$ satisfies $\hat{K} \simeq \bigoplus_{n<\omega}\left[\oplus_{\xi<\alpha_{n}} Z\left(p_{n}\right)\right]$ and hence its homomorphic image $\hat{H}$ satisfies $\hat{H} \simeq \bigoplus_{n<\omega}\left[\oplus_{\xi<\alpha_{n}} F(n, \xi)\right]$ where for each $(n, \xi)$ either $F(n, \xi)=Z\left(p_{n}\right)$ or $F(n, \xi) \stackrel{(n<\infty}{=}\{0\}$. Since $F(n, \xi) \hat{}=F(n, \xi)$ we have

$$
H \simeq \hat{\hat{H}} \simeq \prod_{n<\omega}\left[\prod_{\xi<\alpha_{n}} F(n, \xi)\right]
$$

so that $H$ is (isomorphic to) a product of finite cyclic groups. It is then clear that $t(H)$ is dense in $H$, as required.

(b) $\Rightarrow$ (a). Since (a) is clear when $\alpha=\omega$, we assume here that $\alpha>\omega$. As is well-known [28] (7.6), for $e \neq x \in K$ there is an open, normal subgroup $N(x)$ of $G$ such that $x \notin N(x)$. This furnishes a continuous isomorphism $\varphi$ from $K$ onto a closed subgroup of $\Pi_{x} K / N(x)$, with each $|K / N(x)|<\omega$. Noting that up to topological isomorphism there exist just countably many finite topological groups, say $\left\{F_{n}: n<\omega\right\}$, and identifying $K$ with its image $\varphi[K]$, we have for suitably chosen cardinal numbers $\gamma_{n}$ the topological group inclusion

$$
K \subset F=\prod_{n} F_{n}^{\gamma_{n}} .
$$

Let $\pi_{n}$ be the projection from $F$ onto $F_{n}^{\gamma_{n}}$, and set $Q_{n}=\pi_{n}[K]$ and $\alpha_{n}=w\left(Q_{n}\right)$. Since a continuous function defined on a compact space cannot raise weight [18] (3.7.19), we have $\alpha_{n} \leq \alpha$; and from $K \subset \Pi_{n} Q_{n}$ we have

$$
\alpha=w(K) \leq w\left(\prod_{n} Q_{n}\right) \leq \sum_{n} \alpha_{n}
$$

and hence $\alpha=\sum_{n} \alpha_{n}$.

We claim next that $2^{\alpha_{n}}<2^{\alpha}$ for all $n<\omega$. Indeed if some $N<\omega$ satisfies $2^{\alpha_{N}}=2^{\alpha}$ and $G$ is a small totally dense subgroup of $K$ then since 
$\pi_{N}[G]$ is totally dense in $Q_{N}$ and $Q_{N}=t\left(Q_{N}\right)$ we have from 2.1(a) and 5.2 that

$$
|G| \geq\left|\pi_{N}(G)\right| \geq\left|Q_{N}\right|=2^{\alpha_{N}}=2^{\alpha}=|K|,
$$

a contradiction. Thus $2^{\alpha_{n}}<2^{\alpha}$, and hence $\alpha_{n}<\alpha$, for all $n<\omega$. The condition $\operatorname{cf}(\alpha)=\omega$ now follows from $\alpha=\Sigma_{n} \alpha_{n}$, while $\alpha=\log \left(2^{\alpha}\right)$ is given by 5.4 .

(b) $\Rightarrow$ (c). This is obvious.

(c) $\Rightarrow$ (b). Let $G$ be a small, totally dense subgroup of $K$.

It is well-known and easy to prove, using the fact that weight equals local weight (that is: character) in compact groups, that $w(K) \leq$ $w(C) \cdot w(K / C)$ for every closed normal subgroup $C$ of $K$. In the present case, taking for $C$ the component of the identity in $K$, we have $w(K / C)=\alpha$. For otherwise $w(C)=\alpha$ and from

$$
|G \cap C| \leq|G|<|K|=2^{\alpha}=|C|
$$

it would follow that $G \cap C$ is a small, totally dense subgroup of the connected group $C$, contrary to 5.6. ) Now by 5.3 the canonical homomorphism $\varphi$ from $K$ onto $K / C$ takes $G$ to a totally dense subgroup of $K / C$ such that

$$
|\varphi[G]| \leq|G|<|K|=2^{\alpha}=2^{w(K / C)}=|K / C|,
$$

and the proof is complete.

6. Small, totally dense, pseudocompact subgroups. Here we recapitulate the cardinality constraints of the preceding sections with a view to determining the existence of such subgroups. As was indicated above in our Abstract this question, which was raised in [13] and which provoked the present investigation, cannot be settled on the basis of the axioms of ZFC.

6.1. TheOREM. Assume GCH. No compact topological group has a small, totally dense, pseudocompact subgroup.

Proof. Let $K$ be a counterexample and let $\alpha=w(K)$. Since $\alpha \geq \omega$ we have $\operatorname{cf}(\alpha)=\omega$ from 5.8, so there is a sequence $\left\{\alpha_{n}: n<\omega\right\}$ of cardinals such that each $\alpha_{n}<\alpha$ and $\alpha_{n} \uparrow \alpha$. From GCH we have

$$
2^{\alpha_{n}}=\left(\alpha_{n}\right)^{+} \leq \alpha_{n+1}<\alpha
$$

and hence $\alpha=\log (\alpha)$, so from

$$
2^{\alpha}=2^{\sum \alpha_{n}}=\prod 2^{\alpha_{n}} \leq \alpha^{\omega} \leq 2^{\alpha}
$$

follows $2^{\alpha}=(\log (\alpha))^{\omega}$. But $\mathrm{GCH}$ implies $\mathrm{SCH}$, so from $2.7(\mathrm{e})$ we have $m(K)=|K|$-i.e., $K$ has no small, dense, pseudocompact subgroup. 
6.2. THEOREM. It is consistent with the axioms of ZFC that there exists a compact topological group with a small, totally dense, pseudocompact subgroup; in fact this condition is compatible with $\mathrm{SCH}$.

Proof. It is enough to demonstrate the consistency of the existence of a compact group $K$ which contains a small totally dense subgroup $G_{0}$ and a small, dense, pseudocompact subgroup $G_{1}$; for then (as is clear from the definitions of the concepts in question) the subgroup $G$ of $K$ generated by $G_{0} \cup G_{1}$ is small, totally dense and pseudocompact.

According to 2.7(d) and 5.8, it is enough to arrange for a cardinal $\alpha$ such that $\operatorname{cf}(\alpha)=\omega$ and $\alpha=\log \left(2^{\alpha}\right)$ and $(\log (\alpha))^{\omega}<2^{\alpha}$. The theorem of Easton [17] asserts, roughly speaking, that any phenomenon concerning exponentiation not obviously interdicted by König's theorem is compatible with ZFC. In particular we may choose $\beth_{\xi}<\alpha<\beth_{\xi+1}$ so that $\operatorname{cf}(\alpha)=\omega$ and with $\alpha_{n}$ chosen so that $\alpha_{n} \uparrow \alpha$ and in addition $2^{\alpha_{n}} \uparrow$. (Write $\beta=\sum_{n} 2^{\alpha_{n}}$. From König's theorem and $\mathrm{SCH}$ follows $\beta^{\omega}=\beta^{+}$, so from 5.5(a) we have

$$
2^{\alpha}=\prod 2^{\alpha_{n}} \leq \beta^{\omega}=\beta^{+} \leq 2^{\alpha}
$$

and hence $\beta^{+}=2^{\alpha}$.) Schematically, then,

$$
\beth_{\xi}<\alpha_{n} \uparrow \alpha<\beth_{\xi+1}<2^{\alpha_{n}} \uparrow \beta<\beta^{+}=2^{\alpha} .
$$

From this configuration the three conditions $\operatorname{cf}(\alpha)=\omega, \alpha=\log \left(2^{\alpha}\right)$, and $(\log (\alpha))^{\omega}<2^{\alpha}$ are obvious.

For a specific example, as in [4] (§3), assume $\beth_{1}<\aleph_{\omega}<\beth_{2}$ and $2^{\aleph_{n}}<2^{\aleph_{n+1}}$ for all $n<\omega$, and set $\alpha=\aleph_{\omega}$. For (sufficiently large) $n$ we have $\beth_{1}<\aleph_{n}=\alpha_{n}$ and $\beth_{2}<2^{\aleph_{n}}$, as required.

In summary, we have this result.

6.3. TheOREM. (a) The following two questions are undecidable in ZFC.

(1) Is there a compact group with a small, totally dense, pseudocompact subgroup?

(2) Is there a compact, totally disconnected Abelian group with a small, totally dense, pseudocompact subgroup?

(b) In any model of ZFC, the answer to question (1) is "Yes" if and only if the answer to question (2) is "Yes".

Proof. Statement (a) follows from 6.1 and 6.2, and (b) is immediate from 5.8. 


\section{REFERENCES}

[1] Heinz Bachmann, Transfinite Zahlen, Second Edition, Ergebnisse der Mathematik und ihrer Grenzgebiete, vol. 1, Springer-Verlag, Berlin-Heidelberg-New York, 1967.

[2] R. W. Bagley, E. H. Connell, and J. D. McKnight, Jr., On properties characterizing pseudo-compact spaces, Proc. Amer. Math. Soc., 9 (1958), 500-506.

[3] Bohuslav Balcar and František Franěk, Independent families in complete Boolean algebras, Trans. Amer. Math. Soc., 274 (1982), 607-618.

[4] F. S. Cater, Paul Erdős and Fred Galvin, On the density of $\lambda$-box products, General Topology and its Appl., 9 (1978), 307-312.

[5] W. W. Comfort, Topological groups. In: Handbook of General Topology, edited by Kenneth Kunen and Jerry Vaughan, pp. 1143-1263. North-Holland Publ. Co., Inc., Amsterdam, 1984.

[6] W. W. Comfort and Douglass L. Grant, Cardinal invariants, pseudocompactness and minimality: some recent advances in the topological theory of topological groups, Topology Proceedings, 6 (1981), 227-265.

[7] W. W. Comfort and G. L. Itzkowitz, Density character in topological groups, Mathematische Annalen, 226 (1977), 223-227.

[8] W. W. Comfort and S. Negrepontis, Homeomorphs of three subspaces of $\beta N \backslash N$, Math. Zeitschrift, 107 (1968), 53-58.

[9] _ The Theory of Ultrafilters, Grundlehren der mathematischen Wissenschaften vol. 211, Springer-Verlag, Berlin-Heidelberg-New York, 1974.

[10] W. W. Comfort and Lewis C. Robertson, Compact groups: finer topologies and small dense subgroups, Abstracts Amer. Math. Soc., 2 (1981), 428 [Abstract 788-22-90].

[11] W. W. Comfort and Kenneth A. Ross, Pseudocompactness and uniform continuity in topological groups, Pacific J. Math., 16 (1966), 483-496.

[12] W. W. Comfort and Victor Saks, Countably compact groups and finest totally bounded topologies, Pacific J. Math., 49 (1973), 33-44.

[13] W. W. Comfort and T. Soundararajan, Pseudocompact group topologies and totally dense subgroups, Pacific J. Math., 100 (1982), 61-84.

[14] Keith I. Devlin and R. B. Jensen, Marginalia to a theorem of Silver. In: Proc. International Summer Institute and Logic Colloquium (Kiel, 1974), Lecture Notes in Mathematics no. 499, pp. 115-142, Springer-Verlag, Berlin-Heidelberg-New York, 1975.

[15] Eric K. van Douwen, Homogeneity of $\beta G$ if $G$ is a topological group, Colloq. Math., 41 (1979), 193-199.

[16] _ The weight of a pseudocompact (homogeneous) space whose cardinality has countable cofinality, Proc. Amer. Math. Soc., 80 (1980), 678-682.

[17] William B. Easton, Powers of the regular cardinals, Annals of Math. Logic, 1 (1970), 139-178.

[18] Ryszard Engelking, General Topology, Polska Akademia Nauk, Monographie Matematyczne volume 60. Panstwowe Wydawnictwo Naukowe-Polish Scientific Publishers, Warszawa, 1977.

[19] J. Gerlits, On subspaces of dyadic compacta, Studia Scientarum Math. Hungarica, 11 (1976), 115-120.

[20] Continuous functions on products of topological spaces, Fundamenta Math., 106 (1980), 67-75.

[21] On a generalization of dyadicity, Studia Scientarum Math. Hungarica, 13 $(1978 / 81), 1-17$.

[22] Leonard Gillman and Meyer Jerison, Rings of Continuous Functions, D. Van Nostrand Co., Inc. Princeton-Toronto-London-New York, 1960. 
[23] D. L. Grant, Topological groups which satisfy an open mapping theorem, Pacific J. Math., 68 (1977), 411-423.

[24] _ Arbitrary powers of the roots of unity are minimal Hausdorff topological groups, Topology Proceedings, 4 (1979), 103-108.

[25] Paul R. Halmos, Measure Theory, D. Van Nostrand Co., Inc. New York, 1950.

[26] S. Hartman and A. Hulanicki, Sur les ensembles denses de puissance minimum dans les groupes topologiques, Colloquium Math., 6 (1958), 187-191.

[27] Edwin Hewitt, Rings of real-valued continuous functions I, Trans. Amer. Math. Soc., 64 (1948), 45-99.

[28] Edwin Hewitt and Kenneth A. Ross, Abstract Harmonic Analysis, Volume I, Grundlehren der math. Wissenschaften volume 115, Springer-Verlag, Berlin-GöttingenHeidelberg, 1963.

[29] _ Abstract Harmonic Analysis. Volume II. Grundlehren der math. Wissenschaften volume 152, Springer-Verlag, New York-Heidelberg-Berlin, 1970.

[30] G. Hochschild, The Structure of Lie Groups, Holden-Day, Inc. San FranciscoLondon-Amsterdam, 1965.

[31] Gerald L. Itzkowitz, Extensions of Haar measure for compact connected Abelian groups, Bull. Amer. Math. Soc., 71 (1965), 152-156.

[32] _ Extensions of Haar measure for compact connected Abelian groups, Indag. Math., 27 (1965), 190-207.

[33] _ On the density character of compact topological groups, Fundamenta Math., 75 (1972), 201-203.

[34] L. N. Ivanovskiǐ, On a hypothesis of P. S. Alexandroff, Doklady Akad. Nauk SSSR N.S., 123 (1958), 785-786.

[35] Thomas Jech, Set Theory, Academic Press, New York-London, 1978.

[36] I. Juhász, Cardinal Functions in Topology-Ten Years Later, Mathematical Centre Tracts 123. Mathematisch Centrum, Amsterdam, 1980.

[37] Shizuo Kakutani, On cardinal numbers related with a compact Abelian group, Proc. Imperial Acad. Tokyo, 19 (1943), 366-372.

[38] Shizuo Kakutani and Kunihiko Kodaira, Über das Haarsche Mass in der lokal bikompakten Gruppe, Proc. Imperial Acad. Tokyo, 20 (1944), 444-450.

[39] M. A. Khan, The existence of totally dense subgroups in LCA groups, Pacific J. Math., 112 (1984), 383-390.

[40] J. König, Zum Kontinuumproblem, Mathematische Annalen, 60 (1904), 177-180.

[41] V. Kuz' minov, On a hypothesis of P.S. Alexandroff in the theory of topological groups, Doklady Akad. Nauk SSSR N.S., 125 (1959), 727-729.

[42] Menachem Magidor, On the singular cardinals problem I, Israel J. Math., 28 (1977), $1-31$.

[43] - On the singular cardinals problem II, Annals. of Math., 106 (1977), 517-547.

[44] Victor Saks, Ultrafilter invariants in topological spaces, Trans. Amer. Math. Soc., 241 (1978), 79-97.

[45] B. Ė. Šapirovskiŭ, On imbedding extremally disconnected spaces in compact Hausdorff spaces. b-points and weight of pointwise normal spaces, Doklady Akad. Nauk SSSR, 223 (1975), 1083-1086. [In Russian. English translation: Soviet Math. Doklady 16 (1975), 1056-1061.]

[46] T. Soundararajan, Totally dense subgroups of topological groups. In: General Topology and Its Relations to Modern Analysis and Algebra III, Proc. (1968) Kanpur Topological Conference, pp. 299-300. Academia Press, Prague, 1971.

[47] L. J. Sulley, $A$ Note on $B$ - and $B_{r}$-complete topological abelian groups, Proc. Cambridge Phil. Soc., 66 (1969), 275-279.

[48] Alfred Tarski, Quelques théorèmes sur les alephs, Fundamenta Math., 7 (1925), 1-14. 
[49] N. Th. Varopoulos, $A$ theorem on the continuity of homomorphisms of locally compact groups, Proc. Cambridge Phil. Soc., 60 (1964), 449-463.

[50] N. Ya. Vilenkin, On the dyadicity of the group space of bicompact commutative groups, Uspehi Mat. Nauk N.S., 13:6(84) (1958), 79-80. [In Russian.]

[51] J. de Vries, Pseudocompactness and the Stone-Čech compactification for topological groups, Nieuw Archief voor Wiskunde, (3) 23 (1975), 35-48.

[52] B. L. van der Waerden, Stetigkeitssätze für halbeinfache Liesche Gruppen, Math. Zeitschrift, 36 (1933), 780-786.

[53] André Weil, Sur les Espaces à Structure Uniforme et sur la Topologie Générale, Publ. Math. Univ. Strasbourg. Hermann \& Cie., Paris, 1937.

[54] _ L'intégration dans les Groupes Topologiques et ses Applications, Actualités Scientifiques et Industrielles, Publ. Math. Inst. Strasbourg, Hermann \& Cie., Paris, 1951.

[55] Robert F. Wheeler, On separable z-filters, General Topology and its Appl., 5 (1975), 333-345.

[56] Howard J. Wilcox, Pseudocompact groups, Pacific J. Math., 19 (1966), 365-379.

[57] Howard J. Wilcox, Dense subgroups of compact groups, Proc. Amer. Math. Soc., 28 (1971), 578-580.

Received October 26, 1983 and in revised form January 29, 1984.

WESLEYAN UNIVERSITY

MidDlETOWN, CT 06457 



\section{PACIFIC JOURNAL OF MATHEMATICS EDITORS}

\author{
V. S. VARADARAJAN (Managing Editor) \\ University of California \\ Los Angeles, CA 90024 \\ Charles R. DePrima \\ California Institute of Technology \\ Pasadena, CA 91125 \\ R. FInN \\ Stanford University \\ Stanford, CA 94305
}

\section{HeRmanN FlaschKa \\ University of Arizona \\ Tucson, AZ 85721}

Ramesh A. Gangolli

University of Washington

Seattle, WA 98195

ROBION KIRBY

University of California

Berkeley, CA 94720

\section{C. MOORE}

University of California

Berkeley, CA 94720

\section{H. SAMELSON}

Stanford University

Stanford, CA 94305

HAROLD STARK

University of California, San Diego

La Jolla, CA 92093

\section{ASSOCIATE EDITORS}
R. ARENS
E. F. BECKENBACH
B. H. NeumanN
F. Wolf
K. Yoshida (1906-1982)

\section{SUPPORTING INSTITUTIONS}
CALIFORNIA INSTITUTE OF TECHNOLOGY

\author{
UNIVERSITY OF SOUTHERN CALIFORNIA \\ STANFORD UNIVERSITY \\ UNIVERSITY OF HAWAII \\ UNIVERSITY OF TOKYO \\ UNIVERSITY OF UTAH \\ WASHINGTON STATE UNIVERSITY \\ UNIVERSITY OF WASHINGTON
}
UNIVERSITY OF OREGON

\begin{abstract}
UNIVERSITY OF ARIZONA
UNIVERSITY OF BRITISH COLUMBIA

UNIVERSITY OF CALIFORNIA

MONTANA STATE UNIVERSITY

UNIVERSITY OF NEVADA, RENO

NEW MEXICO STATE UNIVERSITY

OREGON STATE UNIVERSITY
\end{abstract}

The Supporting Institutions listed above contribute to the cost of publication of this Journal, but they are not owners or publishers and have no responsibility for its content or policies.

Mathematical papers intended for publication in the Pacific Journal of Mathematics should be in typed form or offset-reproduced (not dittoed), double spaced with large margins. Please do not use built up fractions in the text of the manuscript. However, you may use them in the displayed equations. Underline Greek letters in red, German in green, and script in blue. The first paragraph must be capable of being used separately as a synopsis of the entire paper. In particular it should contain no bibliographic references. Please propose a heading for the odd numbered pages of less than 35 characters. Manuscripts, in triplicate, may be sent to any one of the editors. Please classify according to the scheme of Math. Reviews, Index to Vol. 39. Supply name and address of author to whom proofs should be sent. All other communications should be addressed to the managing editor, or Elaine Barth, University of California, Los Angeles, California 90024.

There are page-charges associated with articles appearing in the Pacific Journal of Mathematics. These charges are expected to be paid by the author's University, Government Agency or Company. If the author or authors do not have access to such Institutional support these charges are waived. Single authors will receive 50 free reprints; joint authors will receive a total of 100 free reprints. Additional copies may be obtained at cost in multiples of 50 .

The Pacific Journal of Mathematics is issued monthly as of January 1966. Regular subscription rate: $\$ 190.00$ a year (5 Vols., 10 issues). Special rate: $\$ 66.00$ a year to individual members of supporting institutions.

Subscriptions, orders for numbers issued in the last three calendar years, and changes of address should be sent to Pacific Journal of Mathematics, P.O. Box 969, Carmel Valley, CA 93924, U.S.A. Old back numbers obtainable from Kraus Periodicals Co., Route 100, Millwood, NY 10546.

The Pacific Journal of Mathematics at P.O. Box 969, Carmel Valley, CA 93924 (ISSN 0030-8730) publishes 5 volumes per year. Application to mail at Second-class postage rates is pending at Carmel Valley, California, and additional mailing offices. Postmaster: Send address changes to Pacific Journal of Mathematics, P.O. Box 969, Carmel Valley, CA 93924.

PUBLISHED BY PACIFIC JOURNAL OF MATHEMATICS, A NON-PROFIT CORPORATION

Copyright $\odot 1985$ by Pacific Journal of Mathematics 


\section{Pacific Journal of Mathematics}

Vol. 119, No. $2 \quad$ June, 1985

Mustafa Agah Akcoglu and Meira Falkowitz (Soshniak), A general local

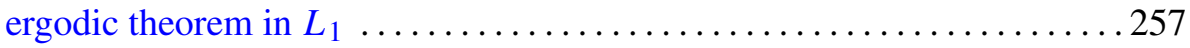

W. Wistar (William) Comfort and Lewis Chandlee Robertson,

Cardinality constraints for pseudocompact and for totally dense

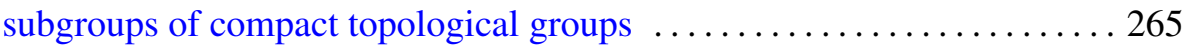

John Morse Delaurentis and Boris G. Pittel, Random permutations and

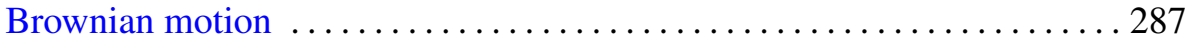

José Esteban Galé, Gel'fand theory in algebras of differentiable functions on Banach spaces ......................................... 303

Harry Gingold, On the location of zeroes of oscillatory solutions of

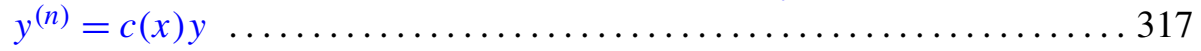

Kei Ji Izuchi, Zero sets of interpolating Blaschke products .............337

Mahesh Nerurkar, Ergodic continuous skew product actions of amenable

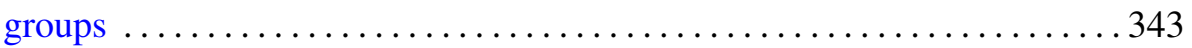

R. Owens, A maximal function characterization of a class of Hardy

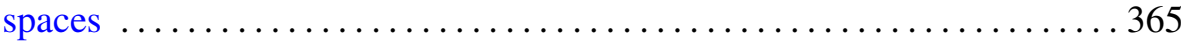

Judith Anne Packer, Point spectrum of ergodic abelian group actions and the corresponding group-measure factors $\ldots \ldots \ldots \ldots \ldots \ldots \ldots \ldots \ldots \ldots \ldots \ldots$

Judith Anne Packer, On the embedding of subalgebras corresponding to quotient actions in group-measure factors $\ldots \ldots \ldots \ldots \ldots \ldots \ldots \ldots . \ldots 4$

Iain Raeburn and Joseph L. Taylor, The bigger Brauer group and étale cohomology

David Rosen, The Diophantine equation $a x+b y=c$ in $Q(\sqrt{5})$ and other number fields

Mau-Hsiang Shih and Kok Keong Tan, Noncompact sets with convex sections

Lee Barlow Whitt, Codimension two isometric immersions between Euclidean spaces

Rodney Ian Yager, Iwasawa theory for the anticyclotomic extension 